\title{
Mating-Type Distribution and Genetic Diversity of Cercospora sojina Populations on Soybean from Arkansas: Evidence for Potential Sexual Reproduction
}

\author{
Hun Kim, Annakay D. Newell, Robyn G. Cota-Sieckmeyer, John C. Rupe, Ahmad M. Fakhoury, and Burton H. Bluhm
}

First, second, third, fourth, and sixth authors: Department of Plant Pathology, University of Arkansas, Fayetteville 72701; and fifth author: Department of Plant, Soil and Agricultural Systems, Southern Illinois University-Carbondale, Carbondale 62901.

Accepted for publication 12 April 2013.

\begin{abstract}
Kim, H., Newell, A. D., Cota-Sieckmeyer, R. G., Rupe, J. C., Fakhoury, A. M., and Bluhm, B. H. 2013. Mating-type distribution and genetic diversity of Cercospora sojina populations on soybean from Arkansas: Evidence for potential sexual reproduction. Phytopathology 103:10451051.

Cercospora sojina causes frogeye leaf spot of soybean, which can cause serious economic losses in the United States. In this study, $132 C$. sojina isolates were collected from six fields (from two counties, Cross and Crawford) in Arkansas. To determine mating type, a multiplex polymerase chain reaction assay was developed with primers specific for C. sojina. Of the 132 isolates, 68 isolates had the MAT1-1-1 idiomorph and 64 isolates had the MAT1-2 idiomorph; no isolates possessed both idiomorphs. Both mating types were present in a variety of spatial scales, including separate lesions on individual leaves. Clone-corrected data from eight microsatellites indicated that mating-type loci were present in

approximately equal proportions in all populations analyzed, which suggests that Arkansas populations of $C$. sojina are undergoing cryptic sexual reproduction. All six populations evaluated had high genotypic diversity of 26 to $79 \%$. In addition, among strains isolated from a single leaf, multiple and distinct haplotypes were associated with both mating types, supporting the hypothesis that sexual reproduction occurs within the populations. Most populations showed significant gametic disequilibrium but levels of disequilibrium were relatively low, particularly in populations from Crawford County. A low differentiation index $\left(G_{S T}\right)$ was observed for all simple-sequence repeat markers across all populations. Furthermore, the value of $G$ statistics between populations suggests that significant genetic exchange exists among the populations. Taken together, these results demonstrate that $C$. sojina populations from Arkansas are genetically diverse and most likely undergoing sexual reproduction.
\end{abstract}

Frogeye leaf spot (FLS), caused by Cercospora sojina Hara, is an economically important disease of soybean in the United States (27). FLS was first described in Japan in 1915 (25), and the first report of $C$. sojina in the United States was in South Carolina in 1924 (25). For many decades, FLS has been prevalent in southern U.S. states $(37,38)$ and, more recently, has become endemic throughout the U.S. Midwest and upper Midwest $(26,42)$. Beyond the United States, C. sojina has been reported in at least 27 countries spanning North and South America, Europe, Africa, and Asia (6).

Symptoms of FLS include circular or angular lesions of 1 to $5 \mathrm{~mm}$ in diameter that initially resemble dark, water-soaked spots (13). As the disease progresses, lesions enlarge into distinctive brown spots with reddish-brown margins, which can coalesce and cause severe leaf blighting or defoliation. C. sojina overwinters in infested soybean residue and can survive in heavily infected seed (27). The disease cycle of FLS is initiated by spores originating from infected leaf debris or lesions on cotyledons resulting from planting heavily infected seed (27). Lesions may not appear until $\approx 2$ weeks after invasion of tissue and, therefore, are not typically observed on young leaves (27). However, conidia may appear as early as $48 \mathrm{~h}$ after inoculation on plants exposed to favorable temperatures $\left(25\right.$ to $\left.30^{\circ} \mathrm{C}\right)$ and high relative humidity $(>90 \%)$. Conidia are carried by air currents and rain splash and cause

Corresponding author: B. H. Bluhm, E-mail address: bbluhm@uark.edu

http://dx.doi.org/10.1094/PHYTO-09-12-0229-R

(c) 2013 The American Phytopathological Society secondary infections throughout the growing season when environmental conditions are favorable. Because FLS is a polycyclic disease, symptoms can increase continuously throughout the growing season and reach epidemic proportions when environmental conditions are favorable or suitable control measures are not employed. Regarding the latter, a serious concern regarding FLS management is the recent emergence of resistance to strobilurin fungicides among U.S. populations of $C$. sojina (45). The rate and mechanism of spread of fungicide resistance throughout populations of $C$. sojina have considerable implications for FLS management, yet are poorly understood.

The potential role of sexual reproduction among populations of $C$. sojina in FLS epidemiology and management has not been established. In many plant-pathogenic fungi, sexual reproduction is a key mechanism through which genetic diversity is produced, and often allows fungal pathogens to overcome host resistance by generating more fit genotypes through recombination (11). However, for most Cercospora spp., including $C$. sojina, a sexual stage has not been observed in either field or laboratory conditions. Based on molecular analyses, Cercospora spp. form a monophyletic group within the teleomorphic genus Mycosphaerella (68,12), and Mycosphaerella teleomorphs have been found for a few Cercospora spp. $(9,39)$. Like many other heterothallic ascomycetes, completion of the sexual cycle in Cercospora spp. is predicated on the presence and interaction of individuals with different idiomorphs, namely MATI-1 and MAT1-2, at a single mating-type locus $(5,14)$. Although the distribution of matingtype idiomorphs throughout natural populations of a given fungus neither proves nor disproves the existence of a sexual stage, equal 
proportions of mating type idiomorphs are expected in populations undergoing sexual reproduction (28).

In the absence of a known sexual stage, several approaches can provide evidence of cryptic sexual reproduction, including measurements of genetic diversity, population differentiation, and mating-type frequencies (28). Populations undergoing sexual reproduction typically have high genetic diversity and equal mating-type frequencies compared with populations solely or predominantly reproducing asexually (28). Recent reports by Groenewald et al. $(15,17)$ showed that at least some populations of $C$. zeae-maydis, C. zeina, and $C$. beticola have $\approx 1: 1$ distributions of mating type idiomorphs, and that populations of $C$. beticola have high levels of genetic diversity. Based on these results, the authors postulated that $C$. beticola populations undergo sexual recombination even though no teleomorph has yet been observed, and that other species of Cercospora may also undergo cryptic sexual reproduction.

In the present study, our overall goal was to assess whether populations of $C$. sojina isolated from Arkansas undergo sexual reproduction. The specific objectives of this study were to (i) develop multiplex polymerase chain reaction (PCR) conditions for rapid identification of mating types in $C$. sojina and use this assay to determine the frequencies and distributions of the two mating types, (ii) determine the level of genetic diversity and population differentiation between and within populations based on microsatellite analyses, and (iii) assess the possibility of sexual reproduction in field populations with multilocus analyses of population structure. To this end, 132 isolates of $C$. sojina were collected from two locations in Arkansas, and the mating type and haplotype of each isolate was defined and analyzed. From this work, a more detailed understanding of the population structure of $C$. sojina has emerged, including evidence for cryptic sexual reproduction.

\section{MATERIALS AND METHODS}

Soybean sampling and fungal isolation. To isolate $C$. sojina from symptomatic soybean tissue, leaves with visible lesions were incubated in a moist chamber (a petri plate containing moist filter paper) at room temperature for 2 to 3 days with a 12-h photoperiod to induce sporulation. Leaves were checked daily for the onset of sporulation with a dissecting microscope. Conidia were collected with a flame-sterilized needle and transferred onto V8 agar medium containing ampicillin $(100 \mu \mathrm{g} / \mathrm{ml})$. The cultures were incubated at room temperature until the colonies were large enough to transfer onto new V8 agar plates. For single-spore isolation, colonies were scraped with a flamed loop and streaked onto fresh V8 agar plates and, subsequently, single isolated colonies were transferred to new V8 agar plates. The plates were incubated for 5 to 7 days after colonies grew visibly. All isolates were stored at $-80^{\circ} \mathrm{C}$ in $20 \%$ glycerol.
Soybean plants showing typical FLS disease symptoms were collected from six fields in two counties (Cross and Crawford) in Arkansas on 11 or 30 September 2009, respectively; these two counties are separated by $\approx 300 \mathrm{~km}$. Four populations (popW1, $n=24$; $p$ opW2, $n=23$; popW3, $n=20$; and popW4, $n=20$ ) were collected from four individual fields near Wynne, AR, in Cross County; each field was separated by roadways which were $\approx 10 \mathrm{~m}$ wide, and five to six diseased plants were collected from each field for $C$. sojina isolation. Additionally, two populations (popK1, $n=21$ and popK2, $n=24$ ) were isolated from two fields of the University of Arkansas Vegetable Research Station near Kibler in Crawford County, and sampling methods were performed as described above. Ultimately, from these six fields, 132 C. sojina isolates were collected for further analysis.

DNA extraction. A $0.5-$ by $-0.5-\mathrm{cm}$ segment of mycelium from each single-spore isolate was collected from cultures grown on V8 agar medium and placed into a $1.5-\mathrm{ml}$ microcentrifuge tube containing $100 \mu \mathrm{l}$ of Tris-EDTA buffer $(10 \mathrm{mM}$ Tris-Cl and $1 \mathrm{mM}$ EDTA, pH 7.5). The tissue was ground in the tube with a sterile micropestle followed by incubation in boiling water for $10 \mathrm{~min}$. After centrifugation, the concentration of genomic DNA in the supernatant was quantified with a ND-1000 spectrophotometer (NanoDrop Technologies, Wilmington, DE).

Multiplex PCR assay for determination of mating types. Degenerate primer pairs MgMfSpMat1-1f1/1r2 and MgMfSpmat12f2/2r1 and Cercospora-specific primer pairs CercosporaMat1f/1r and CercosporaMat2f/2r (Table 1) (14) were used to amplify conserved regions of the MAT1-1-1 and MAT1-2 genes of C. sojina isolates J2L2 and 07-IN. All PCR conditions were followed as described by Groenewald et al. (14). Reaction products were analyzed by electrophoresis in a $1 \%$ (wt/vol) agarose gel with ethidium bromide at $0.1 \mu \mathrm{g} / \mathrm{ml}$ in $1 \times$ Tris-borate-EDTA (TBE) buffer, visualized on a UV transilluminator, and purified with the QIAquick gel extraction kit (Qiagen, Valencia, CA). PCR products were then sequenced with the original primers, and the sequence information was deposited at GenBank. From these sequences, three sets of primers were designed for a multiplex PCR assay: Csojina18Sf/28Sr, CsMat1f/1r, and CsMat2f/2r. Primers were synthesized by Integrated DNA Technologies (Coralville, IA). For each PCR, the total reaction of $50 \mu$ contained $2 \mu$ of genomic DNA, $10 \mu \mathrm{l}$ of $10 \times$ PCR reaction buffer, $2 \mu \mathrm{l}$ of $10 \mathrm{mM}$ dNTPs, $10 \mathrm{pmol}$ of primer sets CsMat1f/1r and CsMat2f/2r, 5 pmol of primer set Csojina18Sf/28Sr, $5 \mu \mathrm{l}$ of $25 \mathrm{mM} \mathrm{MgCl}_{2}$, and 0.7 units of Taq polymerase. The PCR reactions were performed with a 2720 Thermal Cycler (Applied Biosystems, Carlsbad, CA). The initial denaturation step of $94^{\circ} \mathrm{C}$ for 2 min was followed by 40 cycles of amplification $\left(94^{\circ} \mathrm{C}\right.$ for $30 \mathrm{~s}, 58.5^{\circ} \mathrm{C}$ for $30 \mathrm{~s}$, and $72^{\circ} \mathrm{C}$ for $1 \mathrm{~min}$ ), followed by a final elongation step of $72^{\circ} \mathrm{C}$ for $5 \mathrm{~min}$. All PCR products were visualized under UV light on a $1 \%$ (wt/vol) agarose gel containing ethidium bromide at $0.1 \mu \mathrm{g} / \mathrm{ml}$ in $1 \times$ TBE buffer after electrophoretic separation at $95 \mathrm{~V}$ for $1 \mathrm{~h}$.

TABLE 1. Primers used in multiplex polymerase chain reaction (PCR) for identification of mating types

\begin{tabular}{|c|c|c|c|}
\hline Primer & Sequence $\left(5^{\prime}-3^{\prime}\right)$ & Note & Source \\
\hline MgMfSpMat1-1f1 & CATTNGCNCATCCCTTTG & Degenerate primer & 14 \\
\hline MgMfSpMat1-1r2 & GGCTTNGANACCATGGTGAG & Degenerate primer & 14 \\
\hline MgMfSpmat1-2f2 & CAAAGAANGCNTTCNTGATCT & Degenerate primer & 14 \\
\hline MgMfSpmat1-2r1 & TTCTTCTCNGATGGCTTGC & Degenerate primer & 14 \\
\hline CercosporaMat1f & CTTGCAGTGAGGACATGG & Degenerate primer & 14 \\
\hline CercosporaMat1r & GAGGCCATGGTGAGTGAG & Degenerate primer & 14 \\
\hline CercosporaMat $2 \mathrm{f}$ & GATNTACCNTCTCGA & Degenerate primer & 14 \\
\hline CercosporaMat2r & CTGTGGAGCAGTG & Degenerate primer & 14 \\
\hline Csojina18Sf & TCTCCGTAGGTGAACCTGCG & Multiplex PCR primer & This study \\
\hline Csojina28Sr & TATCCCTACCTGATCCGAGGTCAA & Multiplex PCR primer & This study \\
\hline CsMat1f & TGAGGACATGGCCACCCAAATA & Multiplex PCR primer & This study \\
\hline CsMat1r & AAGAGCCCTGTCAAGTGTCAGT & Multiplex PCR primer & This study \\
\hline CsMat2f & TGTTGTAGAGCTCGTTGTTCGCA & Multiplex PCR primer & This study \\
\hline CsMat2r & TCAGACCTTATGAGCTTGAAAGTGCT & Multiplex PCR primer & This study \\
\hline
\end{tabular}


Analysis of genetic diversity. To analyze genetic diversity in each population, we used eight microsatellite (simple sequence repeat [SSR]) markers (Table 2) that showed high levels of polymorphism among a wide range of $C$. sojina isolates (29). SSR loci were amplified via PCR in $25-\mu$ l reactions containing $100 \mathrm{ng}$ of DNA, $30 \mathrm{nM}$ forward and reverse primers, $0.5 \mathrm{mM}$ dNTPs, $2.5 \mathrm{mM} \mathrm{MgCl}_{2}$, and 0.7 units of Taq polymerase. Cycling parameters consisted of an initial denaturation at $95^{\circ} \mathrm{C}$ for $5 \mathrm{~min}$; followed by 30 cycles of $94^{\circ} \mathrm{C}$ for $30 \mathrm{~s}, 56^{\circ} \mathrm{C}$ for $30 \mathrm{~s}, 72^{\circ} \mathrm{C}$ for $60 \mathrm{~s}$; and extension at $72^{\circ} \mathrm{C}$ for $7 \mathrm{~min}$. Reaction products were separated by electrophoresis in 3\% Metaphor agarose gels and detected by staining with ethidium bromide. A 10-bp DNA ladder (Invitrogen, Carlsbad, CA) was used as a size marker. Consistent with similar studies, this approach allowed resolution of 3 to $4 \mathrm{bp}$ among PCR products (30). Polymorphic DNA bands were scored manually with GenAlex 6 (35) to determine the number of haplotypes and create input file formats required for other analysis tools. The total number of alleles, number of effective alleles, and genetic diversity at each SSR locus were estimated with POPGENE, version 1.32 (43), which also estimates values of Nei's unbiased genetic identity and distance for populations. In addition, POPGENE (43) and GenoDive (24) were used to determine indices of genetic differentiation such as $G_{S T}$ and $G^{\prime \prime}{ }_{S T}$. Genotypic diversity was estimated by the method of Stoddart and Taylor (41), and normalized by dividing $\hat{G}$ by the number of samples in each population, as described by Grunwald et al. (17). Multilocus v1.3b (1) was used to determine the value of $r B a r D$, an alternative measure of the index of association for disequilibrium, and the number of locus pairs in linkage disequilibria was estimated with POPGENE, version 1.32 (43).

\section{RESULTS}

Optimization of multiplex PCR conditions. Although sequences of each mating-type idiomorph are fairly well conserved among many Cercospora spp., primers previously designed from other species inconsistently amplified $C$. sojina mating-type genes. Thus, the mating-type loci of $C$. sojina were sequenced and new primers were designed for rapid, high-throughput assessment of mating types. Degenerate primers (Table 1) were used to amplify portions of the mating-type genes MAT1-1-1 and MAT12 , and PCR-based genome walking was subsequently used to obtain the full-length sequences of both idiomorphs. Sequences of mating-type genes were deposited in the National Center for Biotechnology Information database (MAT1-1-1, GenBank num- ber JX047865 and MAT1-2, GenBank number JX047866), and primers that specifically amplified the mating-type alleles of $C$. sojina were created (CsMat1f/r and CsMat2f/r) (Table 1). Also, a primer pair that amplifies the internal transcribed spacer (ITS) region of rDNA (GenBank accession number AY266156) of $C$. sojina was designed to provide an internal positive control to confirm the presence of amplifiable DNA (Table 1).

Multiplex PCR conditions such as annealing temperatures and primer concentrations were optimized. To determine the optimum temperature for PCR, the primers were tested at annealing temperatures of $57.5,58,58.5,58.8$, and $59^{\circ} \mathrm{C}$. Consequently, $58.5^{\circ} \mathrm{C}$ was determined to be the optimum annealing temperature for amplification based on the intensity of PCR products. Increasing the concentration of primer pairs CsMat $1 \mathrm{f} / \mathrm{r}$ and CsMat $2 \mathrm{f} / \mathrm{r}$ relative to the primers amplifying the ITS region of rDNA provided more equal amplification of the two single-copy loci relative to the multicopy rDNA sequence. After evaluating several ratios of primer concentrations, $10 \mathrm{pmol}$ of CsMat $1 \mathrm{f} / \mathrm{r}$ and CsMat $2 \mathrm{f} / \mathrm{r}$ primer pairs with 5 pmol of primer pairs for rDNA provided the most consistent, even amplification of all three products. With this multiplex PCR assay, the mating type locus of 132 C. sojina isolates was assessed, and a single amplicon of either $406 \mathrm{bp}$ (MAT1-1-1) or 298 bp (MAT1-2) was obtained from each isolate (Fig. 1). None of the isolates contained both idiomorphs. All reactions produced an amplicon of $500 \mathrm{bp}$, confirming the presence of amplifiable DNA from the ITS region (Fig. 1). Thus, the assay provided a robust and reliable tool to score alleles at the mating type locus of $C$. sojina.

Distribution of mating types within populations. Among the 132 C. sojina isolates collected from Arkansas, 68 isolates possessed MAT1-1-1 and 64 isolates possessed MAT1-2, a frequency that does not deviate significantly from a $1: 1$ ratio $\left(\chi^{2}=0.12\right)$ (Table 3). At the level of individual fields, population popW3 and popK2 significantly deviated from the null hypothesis of a $1: 1$

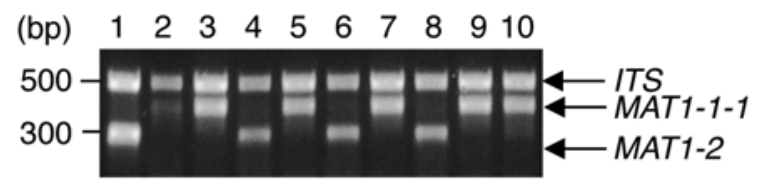

Fig. 1. Multiplex polymerase chain reaction for $M A T$ genes of 10 representative isolates. Lanes 1 (07-IN) and 2 (J2L2) are standard strains for MAT12 and MAT1-1-1, respectively; lanes 3, 5, 7, 9, and 10 show isolates that have MAT1-1-1; and lanes 4, 6, and 8 represent isolates that possess MAT1-2. ITS = internal transcribed spacer.

TABLE 2. Simple-sequence repeat (SSR) markers used to analyze genetic diversity of Cercospora sojina ${ }^{\mathrm{a}}$

\begin{tabular}{|c|c|c|c|c|}
\hline Locus & Primer sequence $\left(5^{\prime}-3^{\prime}\right)^{\mathrm{b}}$ & Repeat motif ${ }^{\mathrm{c}}$ & Size range $(b p)^{d}$ & $N^{\mathrm{e}}$ \\
\hline \multirow[t]{2}{*}{ SSRCs1 } & F: GGTAATCGCCCAGATGAAGA & $(\mathrm{AAC})_{16}$ & $160-185(179)$ & 6 \\
\hline & R: CGAGGAGGTTGGTTGTTGTT & & & \\
\hline SSRCs2 & F: CGCAAAATTTCAGTTCAGCA & $(\mathrm{GAA})_{13}$ & $170-205$ (178) & 5 \\
\hline \multirow[t]{2}{*}{ SSRCs3 } & F: GACGGAATACGGGCTTATGA & $(\mathrm{TTC})_{19}$ & $165-190(171)$ & 4 \\
\hline & R: TCAGAGACTTGTCGCCTCAG & & & \\
\hline \multirow[t]{2}{*}{ SSRCs4 } & F: CCATATTGTCGCCAGGTCTT & $(\mathrm{AGA})_{12}$ & 190-200 (189) & 3 \\
\hline & R: GCTCGCATCTGACATTTCCT & $\ldots$ & & . \\
\hline \multirow[t]{2}{*}{ SSRCs6 } & F: TGGCGAAGACGTCTGATATG & $(\mathrm{TTG})_{13}$ & $215-220(219)$ & 3 \\
\hline & R: TTCTTTCGCATTGCATCAAC & & & \\
\hline \multirow[t]{2}{*}{ SSRCs7 } & F: AAGTATCGGAGCGGACACAG & $(\mathrm{TTCT})_{14}$ & $205-240(235)$ & 3 \\
\hline & R: GTCCAGGCGTTTTAGACGAG & $\ldots$ & & .. \\
\hline \multirow[t]{2}{*}{ SSRCs8 } & F: CCCCATATGCTGCAGAGCTA & $(\mathrm{ATG})_{10}$ & $170-185(171)$ & 3 \\
\hline & R: TCCCTTCTTTGTGCATCATC & $\ldots$ & $\ldots$ & $\ldots$ \\
\hline
\end{tabular}

${ }^{a}$ Originally described by the Molecular Ecology Resources Primer Development Consortium (29).

${ }^{\mathrm{b}} \mathrm{F}$, forward primer; $\mathrm{R}$, reverse primer.

${ }^{c}$ Numbers indicate how many times the repeat occurred in the original sequence.

${ }^{d}$ Numbers in parentheses are the lengths of the alleles derived from the original sequence.

e Observed number of alleles. 
ratio for the two mating type alleles $\left(\chi^{2}=7.2\right.$ and 8.17, respectively) (Table 3). Upon initial consideration, this appeared to indicate a high amount of clonal propagation, especially when considering that the other populations did not deviate significantly from a 1:1 ratio of mating type alleles (Table 3 ). At all locations, we observed that both mating types were frequently detected among isolates collected from distinct lesions on individual leaves. For example, populations popW1, popW2, popW3, and popW4, each of which consisted of 10 individuals isolated from a single leaf, had mating type ratios of 5:5, 5:5, 0:10, and 4:6, respectively (Table 4). However, when clone-corrected data derived from analyses of SSRs were used for the analysis of mating-type distribution, none of the six populations deviated significantly from a 1:1 ratio of mating types (Table 3 ). Thus, the presence of both mating types among populations in close physical proximity (e.g., individual leaves) and the clone-corrected 1:1 mating type frequency throughout spatially distributed populations are consistent with the hypothesis that sexual reproduction is occurring in C. sojina populations in Arkansas.

Genotypic diversity within populations. Various molecular markers such as random amplified polymorphic DNA, amplified fragment length polymorphism, restriction fragment length polymorphism, and microsatellites (SSRs) have been used to analyze genetic diversity of Cercospora spp. $(4,10,15,20,34)$. In populations of $C$. beticola, Groenewald et al. showed the potential of sexual reproduction based on high genotypic diversity and approximately equal proportions of mating-type alleles among populations (15). In addition, the existence of both mating types and different genotypes, as determined by SSR analyses, in individual leaves supports predictions of sexual reproduction in $C$. beticola populations (2). Recently, useful SSR markers were characterized from $C$. sojina (Table 2) (29). In this study, eight SSR loci were analyzed to test the hypothesis that sexual reproduction was occurring in $C$. sojina populations within Arkansas. Among the 132 isolates, 29 alleles were scored at eight SSR loci, and 71 haplotypes were detected. Also, we observed haplotypes that were unique to a single population (12 in popW1, 11 in popW2, 7 in popW3, 6 in popW4, 11 in popK1, and 8 in popK2) and found that few haplotypes were present in multiple populations. For example, only 11 haplotypes were found in at least two populations, and just one haplotype was present in five of the six populations. Values of gene diversity $(H)$ were 0.42 to 0.58 , which were high in all populations (Table 3 ). The popK2 population had the lowest $H$ and the popW1 and popW2 populations had the highest values (Table 4).

Normalized genotypic diversity $(\hat{G} / N)$ was estimated at various spatial scales: individual leaves, fields, and locations (Table 4). Based on analyses of eight polymorphic SSRs, genotypic diversity was observed in various ranges through all populations sampled. Moreover, multiple haplotypes and both mating types were observed among isolates from individual leaves, which suggests that none of the populations were absolutely clonal. The

TABLE 3. Distribution of mating-type genes of Cercospora sojina populations from Arkansas

\begin{tabular}{|c|c|c|c|c|c|c|c|}
\hline \multirow[b]{2}{*}{ Population } & \multirow[b]{2}{*}{ Number of samples } & \multirow[b]{2}{*}{ Clonal fraction $^{\mathrm{a}}$} & \multicolumn{2}{|c|}{ Frequencies $^{\mathrm{b}}$} & \multicolumn{3}{|c|}{$\chi^{2}$ test $^{c}$} \\
\hline & & & MAT1-1-1 & MAT1-2 & Uncorrected data & Clone-corrected data & $P^{\mathrm{d}}$ \\
\hline popW1 & 24 & 0.29 & 0.50 & 0.50 & 0 & 0.06 & 0.81 \\
\hline popW2 & 23 & 0.13 & 0.52 & 0.48 & 0.04 & 0.20 & 0.66 \\
\hline popW3 & 20 & 0.40 & 0.20 & 0.80 & $7.2 *$ & 1.33 & 0.25 \\
\hline popW4 & 20 & 0.35 & 0.40 & 0.60 & 2.98 & 0.08 & 0.78 \\
\hline popK1 & 21 & 0.24 & 0.62 & 0.38 & 1.19 & 1.00 & 0.32 \\
\hline popK2 & 24 & 0.50 & 0.79 & 0.21 & $8.17 *$ & 0.34 & 0.56 \\
\hline Total & 132 & 0.46 & 0.52 & 0.48 & 0.12 & 0.01 & 0.92 \\
\hline
\end{tabular}

${ }^{a}$ Estimated as $1-$ [(number of different genotypes)/(total number of isolates)] (44).

${ }^{\mathrm{b}}$ Frequencies were calculated from uncorrected samples.

c Asterisk $(*)$ indicates mating-type frequencies that are significantly different at $P<0.05$.

${ }^{\mathrm{d}}$ Probability $(P)$ for $\chi^{2}$ of clone-corrected data with 1 degree of freedom.

TABLE 4. Genotypic and genetic diversity within populations at different spatial scales

\begin{tabular}{|c|c|c|c|c|c|c|c|}
\hline Spatial scale, population & $N^{\mathrm{a}}$ & $N_{H}^{\mathrm{b}}$ & $H^{\mathrm{c}}$ & $\widehat{G}^{\mathrm{d}}$ & $\widehat{G} / N(\%)^{\mathrm{e}}$ & $r B a r D^{\mathrm{f}}$ & $L D(\%)^{\mathrm{g}}$ \\
\hline \multicolumn{8}{|l|}{ Within a leaf } \\
\hline popW1 & 10 & 9 & - & 8.3 & 83 & - & - \\
\hline popW2 & 10 & 8 & - & 7.1 & 71 & - & - \\
\hline popW3 & 10 & 4 & - & 1.9 & 19 & - & - \\
\hline popW4 & 10 & 6 & - & 4.5 & 45 & - & - \\
\hline \multicolumn{8}{|l|}{ Within a field } \\
\hline popW1 & 24 & 17 & 0.58 & 12.5 & 52 & $0.204 *$ & $26 / 271(9)$ \\
\hline popW2 & 23 & 20 & 0.58 & 18.2 & 79 & $0.190 *$ & $24 / 279(9)$ \\
\hline popW3 & 20 & 12 & 0.51 & 5.1 & 26 & $0.170 *$ & 17/191 (9) \\
\hline popW4 & 20 & 13 & 0.56 & 10.0 & 50 & $0.301 *$ & $39 / 228(17)$ \\
\hline popK1 & 21 & 16 & 0.43 & 12.6 & 60 & $0.048 * *$ & 12/190 (6) \\
\hline popK2 & 24 & 12 & 0.42 & 6.4 & 27 & $0.137 * *$ & $7 / 207$ (3) \\
\hline \multicolumn{8}{|l|}{ Within a location } \\
\hline popW & 87 & 49 & - & 23.8 & 27 & $0.198 *$ & - \\
\hline popK & 45 & 26 & - & 15.2 & 34 & $0.088 *$ & - \\
\hline
\end{tabular}

a Number of samples in population.

b Number of haplotypes.

c Nei's gene diversity $(H)$ within populations $(31)$; - indicates not calculated.

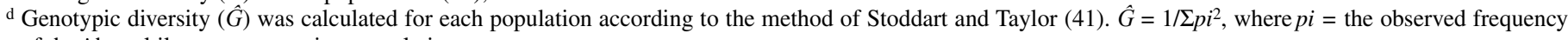
of the $i$ th multilocus genotype in a population.

e Normalized genotypic diversity $(\hat{G} / N)$ was calculated by dividing $\hat{G}$ by the number of samples in each population (17).

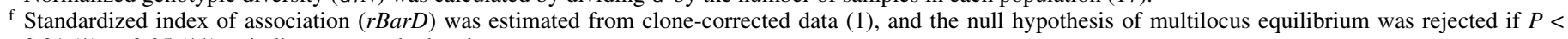
$0.01(*)$ or $0.05(* *) ;-$ indicates not calculated.

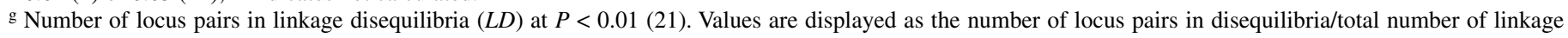
pairs, with the percentage given in parentheses; - indicates not calculated. 
normalized genotypic diversity varied between populations: $\approx 20$ to $80 \%$ at the level of individual leaves and fields (Table 4) as well as at the level of location (popW, Wynne and popK, Kibler).

Tests for multilocus gametic disequilibrium by index of association $(r B a r D)$ showed that all populations were in significant disequilibrium (Table 4). The lowest $r$ BarD value occurred in popK1 (0.048), and both populations from Kibler had relatively low values compared with populations from Wynne, at 0.170 to $0.301(P<0.01)$. In addition, the percentage of locus pairs in significant linkage disequilibrium $\left(\chi^{2}\right.$ test, $\left.P<0.01\right)$ in the six populations ranged from 3\% (7 of 207 pairs) in the popK2 population to $17 \%$ (39 of 228 pairs) in the popW4 population (Table $4)$; at the $P<0.05$ significance level, the percentage was 12 to $24 \%$ (data not shown).

Genetic diversity and differentiation of populations. The contribution of each SSR locus to measurements of genetic diversity varied substantially (Table 5). All eight loci evaluated were polymorphic, with 2 to 5 alleles detected per locus (average $=3.6$ alleles), and the effective number of alleles was 1.6 to 3.2 (Table 5). Among the SSR loci analyzed, SSRCs7 had the highest $H_{T}$ and $H_{S}$ values, and locus SSRCs8 had the lowest (Table 5). The SSR locus SSRCs1 had the highest fixation index $\left(G_{S T}, 0.139\right)$ and SSRCs7 had the lowest fixation index $\left(G_{S T}, 0.040\right)$; the average $G_{S T}$ of all loci was 0.084 (Table 5). The low value of $G_{S T}$ observed in this study was consistent with a previous report of Mycosphaerella graminicola populations retaining sexual reproduction (18), and indicates a relatively high migration rate. Furthermore, when other parameters such as $G_{S T}^{\prime}$ and $G_{S T}^{\prime \prime}$ were used, the values were also relatively low (data not shown).

Pairwise calculations of Nei's unbiased genetic identity between six populations of $C$. sojina in Arkansas were uniformly high (0.851 to 0.991) (Table 6). Intriguingly, the values among the populations collected at Wynne (0.945 to 0.991) were relatively high compared with populations collected at Kibler (0.857) (Table $6)$. In some cases, the genetic identity between populations from different locations was as high as that observed between popu-

TABLE 5. Nei's gene diversity (33) of Cercospora sojina at eight microsatellite (simple sequence repeat [SSR]) loci from clone-corrected samples

\begin{tabular}{llllll}
\hline Locus & $N_{O}{ }^{\mathrm{a}}$ & $N_{E}^{\mathrm{b}}$ & $H_{T}^{\mathrm{c}}$ & $H_{S}^{\mathrm{d}}$ & $H_{S T}{ }^{\mathrm{e}}$ \\
\hline SSRCs1 & 5 & 2.2 & 0.639 & 0.550 & 0.139 \\
SSRCs2 & 5 & 2.2 & 0.587 & 0.549 & 0.065 \\
SSRCs3 & 4 & 2.1 & 0.581 & 0.524 & 0.097 \\
SSRCs4 & 3 & 2.0 & 0.529 & 0.488 & 0.079 \\
SSRCs5 & 3 & 1.9 & 0.521 & 0.479 & 0.081 \\
SSRCs6 & 2 & 1.7 & 0.461 & 0.410 & 0.112 \\
SSRCs7 & 4 & 3.2 & 0.719 & 0.691 & 0.040 \\
SSRCs8 & 3 & 1.7 & 0.445 & 0.417 & 0.062 \\
Mean & 3.6 & 2.1 & 0.560 & 0.514 & 0.084 \\
\hline
\end{tabular}

a Number of alleles in the total samples.

${ }^{\mathrm{b}}$ Effective number of alleles.

c Gene diversity in the total samples over all populations.

d Mean gene diversity averaged over all populations.

e Nei's coefficient of genetic differentiation (32); calculated by $H_{T}-H_{S} / H_{T}$. All values were significant at $P<0.05$. lations from the same location (e.g., popW2/popK2 versus popW2/popW4). The lowest genetic identity (0.851) was found between popW3 and popK1. These results indicate that the majority of genetic diversity is distributed on a small spatial scale in Arkansas. Related to population differentiation, $G^{\prime \prime}{ }_{S T}$ values between populations indicated significant genetic differentiation between approximately two-thirds of the pairwise comparisons; the remaining comparisons were not significant (Table 6 ). Other parameters such as $G_{S T}, G_{S T}^{\prime}$, and Jost's $D$ indicated either low levels of differentiation or nondifferentiation. Overall, these values of differentiation reflect the correlation in genetic identity between populations. Taken together, these results suggest that there has been significant genetic exchange among the populations.

\section{DISCUSSION}

In this study, we developed a multiplex PCR assay to rapidly score mating type loci in $C$. sojina, and investigated genetic diversity and structure of Arkansas populations with polymorphic SSR markers. The populations did not deviate significantly from a $1: 1$ ratio of mating types, and the analysis of SSRs supported the hypothesis that sexual reproduction occurs among populations of C. sojina. Additionally, by providing the first molecular-level perspective of the genetic diversity among populations of $C$. sojina, this study provides important information to guide FLS management strategies, particularly in the context of emerging resistance to strobilurin fungicides, as well as ongoing efforts to improve FLS resistance via conventional breeding approaches.

Analyses of multilocus gametic disequilibrium by index of association $(r B a r D)$ showed that all populations were in significant disequilibrium. However, it is important to note that the presence of gametic disequilibrium within populations of $C$. sojina is not surprising given the current understanding of the FLS disease cycle. The populations analyzed in this study were obtained from soybean fields experiencing high levels of FLS relatively late in the growing season and, thus, asexual reproduction was likely to predominate. Thus, random mating at a specific point in the disease cycle (e.g., the initial or final stage) is the most likely explanation for the simultaneous detection of high levels of genotypic diversity and significant levels of gametic disequilibrium. Consistent with this concept, low index of association values have been observed in fungal populations in which sexual reproduction is postulated to occur at low levels (e.g., C. beticola and Pyrenophora teres f. sp. teres) $(15,36)$. Additionally, populations underlying disease outbreaks may be dominated by closely related individuals, because certain genotypes may be favored by selection pressure associated with environment or prevailing host genotypes (40). Host effects on pathogen diversity are particularly relevant for FLS in the United States, in which monocultures of a relatively limited number of soybean cultivars may dominate a wide geographical area. Thus, during FLS outbreaks, certain genotypes of the pathogen may increase in frequency and generate disequilibrium until sexual recombination has had time to randomize the genetic background.

TABLE 6. Nei's unbiased genetic identity (32) and corrected standardized fixation index (19,23) by pairwise comparisons of field populations

\begin{tabular}{|c|c|c|c|c|c|c|}
\hline \multirow[b]{2}{*}{ Population } & \multicolumn{6}{|c|}{ Genetic identity/fixation index ${ }^{a}$} \\
\hline & popW1 & popW2 & popW3 & popW4 & popK1 & popK2 \\
\hline popW1 & $\ldots$ & 0.991 & 0.950 & 0.979 & 0.882 & 0.863 \\
\hline popW2 & ns & $\ldots$ & 0.945 & 0.984 & 0.885 & 0.933 \\
\hline popW3 & 0.001 & 0.008 & $\ldots$ & 0.991 & 0.970 & 0.851 \\
\hline popW4 & ns & ns & ns & $\ldots$ & 0.918 & 0.917 \\
\hline popK1 & 0.077 & 0.077 & ns & 0.045 & $\ldots$ & 0.857 \\
\hline popK2 & 0.087 & 0.035 & 0.100 & 0.041 & 0.119 & $\ldots$ \\
\hline
\end{tabular}

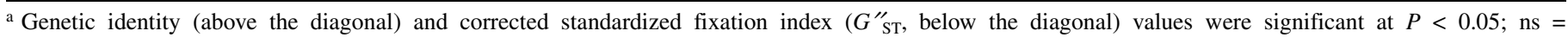
nonsignificant. 
Regarding the linkage disequilibrium observed among locus pairs, a variety of phenomena could explain the levels of disequilibrium detected in this study, including population stratification, genetic drift, and linkage among markers. However, in the absence of a physical or genetic map of the $C$. sojina genome, it is not currently possible to examine the causes of the linkage disequilibrium observed among SSR markers in this study.

Overall, the levels of genotypic diversity observed within each population were consistent with levels of genotypic diversity typically observed in sexually reproducing populations of fungi (28). Particularly in popK1, the low clonal fraction, low gametic disequilibrium, high genotypic diversity, and equal frequencies of the two mating types are consistent with recent or ongoing sexual reproduction. Other populations, in contrast, had intermediate levels of disequilibrium and moderate clonal fractions, most likely resulting from higher levels of asexual reproduction or less outcrossing. The differences observed between populations might be due, in part, to small sample sizes or sampling methods rather than population structures. However, these factors do not preclude the possibility of sexual reproduction, especially considering that a small amount of sexual recombination may be sufficient to sustain high levels of genotypic diversity (22).

The level of genetic diversity observed among populations of C. sojina in Arkansas indicates that sexual reproduction may facilitate rapid adaptation in the pathogen. This finding has significant implications for the management of FLS. First, sexual reproduction presumably provides $C$. sojina a mechanism to overcome host resistance. Consistent with this idea, multiple races of $C$. sojina have been described based on virulence on differential soybean cultivars (25), each of which presumably possesses a distinct set of resistance genes. The high level of genetic diversity identified in this study suggests that many more races of C. sojina are likely to exist or are capable of arising in response to selection pressure and, thus, conventional strategies to breed for resistance should take high levels of diversity into account. For example, sexual recombination is likely to be occurring outside of Arkansas populations of $C$. sojina and, thus, resistance that is robust in one geographical area may not be effective or durable against regionally distinct populations of the pathogen. Second, cryptic sexual recombination in $C$. sojina may accelerate the spread of resistance to strobilurin fungicides. For example, if the initial emergence of strobilurin resistance occurs at a relatively low frequency, resistance would be present in a limited number of haplotypes (and presumably only a few races) in the absence of sexual recombination. Thus, the spread of resistance could be limited by natural constraints on the ability of those specific haplotypes to spread. Sexual recombination, however, could rapidly accelerate the distribution of fungicide resistance throughout populations and into diverse genetic backgrounds of the pathogen. In this scenario, a localized emergence of resistance could conceivably be distributed throughout many races within a few sexual cycles. Resistance to strobilurin fungicides continues to be reported in new locations throughout the United States; therefore, populations of resistant isolates could be evaluated as described in this study to assess the role that sexual reproduction plays in dissemination of resistance.

The results of this study call for additional experimentation to address the potential role of ascospores in the epidemiology of FLS. The $C$. sojina populations analyzed in this study were collected from fields in which plants were approaching maturity and exhibiting large numbers of lesions per leaf, which more accurately approximates the peak of an outbreak rather than its initiation. Based on the levels of genetic diversity detected in this study and the lack of observations of sexual fruiting bodies or ascospores during foliar disease development, it is possible that FLS is initiated, at least in some years or circumstances, by ascospores formed in residual leaf debris from the previous growing season; in this scenario, ascospores could be dispersed locally or over considerable distances, as has been observed for related Mycosphaerella spp. (3). Although the populations analyzed in this study had doubtlessly undergone several cycles of asexual reproduction before sampling occurred, the clone-corrected results still indicated that sexual reproduction was likely to be a driving force underlying the observed population diversity. The extent to which cryptic sexual reproduction is predicted to be occurring among global populations of $C$. sojina could be determined by analyzing populations collected from additional, geographically diverse locations. Moreover, temporal analyses of genetic diversity of $C$. sojina populations throughout the initiation, apex, and conclusion of a disease outbreak could potentially illuminate the timing and importance of sexual reproduction within a growing season.

In this study, eight polymorphic SSR markers, with repeat motifs of three to eight bases (Table 2), were used to analyze populations of $C$. sojina. These markers were sufficiently polymorphic to score on high-resolution agarose gels with conventional electrophoresis rather than more sophisticated analysis platforms, such as capillary electrophoresis. Analyses of SSR markers on agarose gels has the distinct advantages of being inexpensive and widely accessible (30); thus, this approach was selected with the hope of facilitating follow-up studies among members of the U.S. and international FLS research community, in which a wide range of geographically distinct $C$. sojina populations could potentially be analyzed. However, it is possible that a certain level of genetic diversity will be lost with agarose-based analyses due to less resolution of PCR product sizes compared with other methods. We believe that SSR markers with larger repeat motifs, as used in this study, obviate this concern to a considerable extent, evidence of which is the fact that agarosebased analyses of the SSR markers used in this study identified sufficient diversity to strongly support predictions of cryptic sexual reproduction within Arkansas populations of $C$. sojina. As additional genome sequence resources become available for $C$. sojina, the identification and deployment of new SSR markers will doubtlessly contribute to future efforts to study the genetic diversity of the pathogen. Thus, in the future, refined methods of SSR analysis may be required to fully address questions of genetic diversity in $C$. sojina.

Based on the high levels of genetic diversity observed within Arkansas populations of $C$. sojina, new hypotheses regarding the genetic basis of host specificity can be formulated and tested. Many species of Cercospora, including $C$. sojina, have a narrowly restricted host range (6). The genetic and molecular basis of host specificity among Cercospora spp. is a fascinating yet poorly studied phenomenon, and has broad potential implications for the management of Cercospora diseases as well as the refinement of taxonomic relationships within the genus. Historically, host range was a key component of the species concept among Cercospora spp., although recent refinements to the taxonomy of Cercospora spp., including $C$. sojina, have drawn most heavily on morphological characteristics and DNA sequence information at selected loci (16). To date, $C$. sojina has been reported as pathogenic on a handful of species within the Leguminosae family, predominantly of the genera Glycine and Mucuna (6). Given this host range, future experiments could be devised to test whether relationships exist between selected $C$. sojina genotypes and pathogenicity on various plant species reported to be compatible hosts for the pathogen, thus potentially clarifying the importance of host range as a component of the species concept for the organism.

\section{ACKNOWLEDGMENTS}

This research was supported by the United Soybean Board (award number 2262), the Arkansas Soybean Promotion Board, and the University of Arkansas Division of Agriculture. We thank S. Goodwin for guidance during the preparation of the manuscript. 


\section{LITERATURE CITED}

1. Agapow, P. M., and Burt, A. 2001. Indices of multilocus linkage disequilibrium. Mol. Ecol. Notes 1:101-102.

2. Bolton, M. D., Secor, G. A., Rivera, V., Weiland, J. J., Rudolph, K., Birla, K., Rengifo, J., and Campbell, L. G. 2012. Evaluation of the potential for sexual reproduction in field populations of Cercospora beticola from USA. Fungal Biol. 116:511-521.

3. Brunner, P. C., Stefanato, F. L., and McDonald, B. A. 2008. Evolution of the CYP51 gene in Mycosphaerella graminicola: Evidence for intragenic recombination and selective replacement. Mol. Plant Pathol. 9: 305-316.

4. Cai, G., and Schneider, R. W. 2008. Population structure of Cercospora kikuchii, the causal agent of Cercospora leaf blight and purple seed stain in soybean. Phytopathology 98:823-829.

5. Coppin, E., Debuchy, R., Arnaise, S., and Picard, M. 1997. Mating types and sexual development in filamentous ascomycetes. Microbiol. Mol. Biol. Rev. 61:411-428.

6. Crous, P. W., and Braun, U. 2003. Mycosphaerella and its anamorphs: 1. Names published in Cercospora and Passalora. CBS Biodivers. Ser. 1:1571.

7. Crous, P. W., Groenewald, J. Z., Groenewald, M., Caldwell, P., Braun, U., and Harrington, T. C. 2006. Species of Cercospora associated with grey leaf spot of maize. Stud. Mycol. 55:189-197.

8. Crous, P. W., Groenewald, J. Z, Mansilla, J. P., Hunter, G. C., and Wingfield, M. J. 2004. Phylogenetic reassessment of Mycosphaerella spp. and their anamorphs occurring on Eucalyptus. Stud. Mycol. 50:195-214.

9. Deighton, F. C. 1967. New names in Mycosphaerella (M. arachidis and M. pruni-persici) and validation of M. rosicola. Trans. Br. Mycol. Soc. 50:328-329.

10. Dunkle, L. D., and Levy, M. 2000. Genetic relatedness of African and United States populations of Cercospora zeae-maydis. Phytopathology 90:486-490.

11. Glass, L. N., and Kuldau, G. A. 1992. Mating type and vegetative incompatibility in filamentous ascomycetes. Annu. Rev. Phytopathol. 30:201-224.

12. Goodwin, S. B., Dunkle, L. D., and Zismann, V. L. 2001. Phylogenetic analysis of Cercospora and Mycosphaerella based on the internal transcribed spacer region of ribosomal DNA. Phytopathology 91:648-658.

13. Grau, C. R., Dorrance, A. E., Bond, J., and Russin, J. S. 2004. Fungal diseases. Pages 679-763 in Soybeans: Improvement, Production, and Uses. H. R. Boerma and J. E. Specht, eds. ASA, CSSA, and SSSA, Madison, WI.

14. Groenewald, M., Groenewald, J. Z., Harrington, T. C., Abeln, E. C., and Crous, P. W. 2006. Mating type gene analysis in apparently asexual Cercospora species is suggestive of cryptic sex. Fungal Genet. Biol. 43:813-825.

15. Groenewald, M., Linde, C. C., Groenewald, J. Z., and Crous, P. W. 2008. Indirect evidence for sexual reproduction in Cercospora beticola populations from sugar beet. Plant Pathol. 57:25-32.

16. Groenewald, J. Z., Nakashima, C., Nishikawa, J., Shin, H.-D., Park, J.-H., Jama, A. N., Groenewald, M., Braun, U., and Crous, P. W. 2013. Species concepts in Cercospora: Spotting the weeds among the roses. Stud. Mycol. 75:115-170.

17. Grunwald, N. J., Goodwin, S. B., Milgroom, M. G., and Fry, W. E. 2003. Analysis of genotypic diversity data for populations of microorganisms. Phytopathology 93:738-746.

18. Gurung, S., Goodwin, S. B., Kabbage, M., Bockus, W. W., and Adhikari, T. B. 2011. Genetic differentiation at microsatellite loci among populations of Mycosphaerella graminicola from California, Indiana, Kansas, and North Dakota. Phytopathology 101:1251-1259.

19. Hedrick, P. 2005. A standardized genetic differentiation measure. Evolution 59:1633-1638.

20. Imazaki, I., Homma, Y., Kato, M., Vallone, S., Yorinori, J. T., Henning, A. A., Iizumi, H., and Koizumi, S. 2006. Genetic relationships between Cercospora kikuchii populations in South America and Japan. Phytopathology 96:1000-1008.

21. Lee, J.-K., Kim, H., Jeon, J.-J., Kim, H.-S., Zeller, K. A., Carter, L. L. A., Leslie, J. F., and Lee, Y.-W. 2012. Population structure of and mycotoxin production by Fusarium graminearum from maize in South Korea. Appl. Environ. Microbiol. 78:2161-2167.

22. Leslie, J. F., and Klein, K. K. 1996. Female fertility and mating type effects on effective population size and evolution in filamentous fungi. Genetics 144:557-567.

23. Meirmans, P. G., and Hedrick, P. W. 2011. Assessing population structure: $F_{S T}$ and related measures. Mol. Ecol. Resour. 11:5-18.

24. Meirmans, P. G., and Van Tienderen, P. H. 2004. GENOTYPE and GENODIVE: Two programs for the analysis of genetic diversity of asexual organisms. Mol. Ecol. Notes 4:792-794.

25. Melchers, L. E. 1925. Diseases of cereal and forage crops in the United States in 1924. Plant Dis. Rep. (Suppl.) 40:186.

26. Mengistu, A., Kurtzweil, N. C., and Grau, C. R. 2002. First report of frogeye leaf spot (Cercospora sojina) in Wisconsin. Plant Dis. 86:1272.

27. Mian, M. A., Missaoui, A. M., Walker, D. R., Phillips, D. V., and Boerma, H. R. 2008. Frogeye leaf spot of soybean: A review and proposed race designations for isolates of Cercospora sojina Hara. Crop Sci. 48:14-24.

28. Milgroom, M. G. 1996. Recombination and the multilocus structure of fungal populations. Annu. Rev. Phytopathol. 34:457-477.

29. Molecular Ecology Resources Primer Development Consortium, Andris, M., Arias, M. C., Barthel, B. L., Bluhm, B. H., Bried, J., Canal, D., Chen, X. M., Cheng, P., Chiappero, M. B., Coelho, M. M., Collins, A. B., Dash, M., Davis, M. C., Duarte, M., Dubois, M. P., Françoso, E., Galmes, M. A., Gopal, K., Jarne, P., Kalbe, M., Karczmarski, L., Kim, H., Martella, M. B., Mcbride, R. S., Negri, V., Negro, J. J., Newell, A. D., Piedade, A. F., Puchulutegui, C., Raggi, L., Samonte, I. E., Sarasola, J. H., See, D. R., Seyoum, S., Silva, M., Solaro, C., Tolley, K. A., Tringali, M., Vasemägi, A., Xu, L. S., and Zanón-Martínez, J. 2012. Permanent genetic resources added to Molecular Ecology Resources Database 1 February 2012-31 March 2012. Mol. Ecol. Resour. 12:779-781.

30. Morgante, M., Pfeiffer, A., Jurman, I., Paglia, G., and Olivieri, A. M. 2001. PCR analysis of SSR polymorphisms in plants using agarose gels. Pages 206-207 in: Molecular Tools for Screening Biodiversity: Plants and Animals. A. Karp, D. S. Ingram, and P. G. Isaac, eds. Kluwer Academic Publishers, Dordrecht, The Netherlands.

31. Nei, M. 1973. Analysis of gene diversity in subdivided populations. Proc. Natl. Acad. Sci. USA 70:3321-3323.

32. Nei, M. 1978. Estimation of average heterozygosity and genetic distance from a small number of individuals. Genetics 89:583-590.

33. Nei, M. 1987. Molecular Evolutionary Genetics. Columbia Press, New York.

34. Okori, P., Rubaihayo, P. R., Ekwamu, A., Fahleson, J., and Dixelius, C. 2004. Genetic characterization of Cercospora sorghi from cultivated and wild sorghum and its relationship to other Cercospora fungi. Phytopathology 94:743-750

35. Peakall, R., and Smouse, P. E. 2006. GENALEX 6: Genetic analysis in Excel. Population genetic software for teaching and research. Mol. Ecol. Notes 6:288-295.

36. Rau, D., Brown, A. H. D., Brubaker, C. L., Attene, G., Balmas, V., Saba, E., and Papa, R. 2003. Population genetic structure of Pyrenophora teres Drechs., the causal agent of net blotch of barley (Hordeum vulgare L.). Theor. Appl. Genet. 106:947-59.

37. Rosso, M. L., Vazquez, A., and Rainey, K. M. 2011. First report of frogeye leaf spot of soybean caused by Cercospora sojina race 11 in Virginia. Plant Dis. 95:878.

38. Sinclair, J. B., and Backman, P. A. 1989. A Compendium of Soybean Diseases, 3rd ed. American Phytopathological Society, St. Paul, MN.

39. Sivanesan, A. 1985. Teleomorphs of Cercospora sesami and Cercoseptoria sesami. Trans. Br. Mycol. Soc. 85:397-404.

40. Smith, M. J., Feil, E. J., and Smith, N. H. 2000. Population structure and evolutionary dynamics of pathogenic bacteria. BioEssays 22:1115-1122.

41. Stoddart, J. A., and Taylor, J. F. 1988. Genotypic diversity: Estimation and prediction in samples. Genetics 118:705-711.

42. Yang, X. B., Uphoff, M. D., and Sanogo, S. 2001. Outbreaks of soybean frogeye leaf spot in Iowa. Plant Dis. 85:443.

43. Yeh, F. C., Yang, R. C., Boyle, T. B. J., Ye, Z. H., and Mao, J. X. 1997. POPGENE, the User-Friendly Shareware for Population Genetic Analysis. Molecular Biology and Biotechnology Centre, University of Alberta, Canada.

44. Zhan, J., Mundt, C. C., Hoffer, M. E., and McDonald, B. A. 2002. Local adaptation and effect of host genotype on the evolution of virulence: An experimental test in a plant pathosystem. J. Evol. Biol. 15:634-647.

45. Zhang, G. R., Newman, M. A., and Bradley, C. A. 2012. First report of the soybean frogeye leaf spot fungus (Cercospora sojina) resistant to quinone outside inhibitor fungicides in North America. Plant Dis. 96:767. 